\title{
Vaccine research and development: tuberculosis as a global health threat
}

\author{
MOHAMMED MAIKUDI USMAN ${ }^{1,2}$, SALMAH ISMAIL ${ }^{1}$, TEOW CHONG TEOH ${ }^{l}$ \\ ${ }^{1}$ Institute of Biological Sciences, Faculty of Science, University of Malaya, Kuala Lumpur, Malaysia \\ ${ }^{2}$ Department of Biotechnology, School of Pure and Applied Sciences, Modibbo Adama University of Technology, Yola, Nigeria
}

\begin{abstract}
One of the aims of the World Health Organisation (WHO) Millennium Development Goals (MDG) is to reduce the number of cases of tuberculosis (TB) infection by the year 2015. However, 9 million new cases were reported in 2013, with an estimated 480,000 new cases of multi-drug resistant tuberculosis $(M D R-T B)$ globally. Bacille Calmette-Guérin $(B C G)$ is the most available and currently used candidate vaccine against tuberculosis; it prevents childhood TB, but its effectiveness against pulmonary TB in adults and adolescents is disputed. To achieve the goal of the WHO MDG, the need for a new improved vaccine is of primary importance. This review highlights several articles that have reported vaccine development. There are about 16 TB vaccines in different phases of clinical trials at the time of writing, which include recombinant peptide/protein, live-attenuated and recombinant live-attenuated, protein/ adjuvant, viral-vectored, and immunotherapeutic vaccine. Further studies in reverse vaccinology and massive campaigns on vaccination are needed in order to achieve the target for TB eradication by 2050.
\end{abstract}

Key words: tuberculosis, Mycobacterium tuberculosis, vaccine, clinical trials, BCG vaccine.

(Cent Eur J Immunol 2017; 42 (2):196-204)

\section{Introduction}

Tuberculosis is a disease caused by an infectious agent called Mycobacterium tuberculosis (Mtb). Despite reports of declining TB cases in recent years, it also remains a leading deadly infectious disease globally, second only to HIV [1]. In 2012, an estimated 8.7 million new infections in the year 2011, out of which 1.4 million people died of tuberculosis, was published by the WHO. The report also showed highest burden in Asia and Africa, while China and India accounted for almost $40 \%$ of the total TB cases globally [2]. Although there are great achievements globally regarding the threat of TB, in Sub-Saharan Africa tuberculosis remains a major cause of morbidity and mortality [3]. Some factors are responsible for the growth of TB in Africa, the HIV epidemic being the most important one $[4,5]$. An increase in the incidence of MDR-TB is another factor that threatens the efforts towards TB control throughout the world [6]. At latency stage of TB infection the containment of infection is achieved by the host, indicated by survival of mycobacteria in relatively stable numbers [7]. Patients with latent tuberculosis typically do not feel ill and are not infectious [8].

World Health Organisation (WHO) Millennium Development Goals (MDG) are a set of targets aimed at expressing key points of human development [9]. The goals of MDG were generated at the United Nations Millennium
Summit in September 2000 [10]. In addition to a target associated with MDG and endorsed by the Stop TB partnership for reducing its prevalence and death as a result by $50 \%$, the target was also aimed at eradication of TB as a worldwide health threat by the year 2050 [11].

Bacille Calmette-Guérin is a vaccine against tuberculosis, developed in 1921 by Albert Calmette and Camille Guérin, caused by attenuation of the Mycobacterium bovis strain [12]. The novel tuberculosis vaccines need to be better in efficacy and safety, or both, than BCG. Therefore, the urgent need for alternative anti-tuberculosis vaccines is of paramount importance. Bacille Calmette-Guérin immunisation is still in used because of the protection it gives against the infant form of tuberculosis [13, 14]. Administration of diverse booster vaccines later to BCG prime strengthens the protection induced by BCG. Also, other vaccines serve as replacements to Bacille Calmette-Guérin for the generation of a superior immune response up-front. In both cases, the aim of vaccination is the generation of long lasting protection against the most prevalent form of pulmonary tuberculosis in all age groups $[13,14]$.

Complete-genome sequencing, and comparative and system biology lead to new knowledge into the origin and evolution of $M t b$ and the molecular principle of its pathogenicity. These have vital implications about our perspective of the new vaccine development [15]. Progress in the fields of molecular biology, genomics, proteomics, and

Correspondence: Prof. Salmah Ismail, Institute of Biological Sciences, Faculty of Science, University of Malaya, 50603 Kuala Lumpur,

Malaysia, e-mail: salmah_r@um.edu.my

Submitted: 12.11.2015; Accepted: 10.11.2016 
transcriptomics contributed tremendously in the search for a new and enhanced tuberculosis vaccines [16]. Some TB vaccines have entered different stages of clinical trials in recent years. An attempt to review recent research and development of TB vaccines is given below.

\section{Disease burden of tuberculosis}

Tuberculosis is likely to remain in a position of a major public health problem in the coming decades because of its large global load [17]. According to a 2014 (WHO) report on TB, an estimated figure of 1.5 million deaths were recorded in 2013 (0.4 million were HIV-infected people and 1.1 million were HIV-negative) [18]. $M t b$ and HIV infections act in a collaborative manner [19]. The role of CD4 T cells is of paramount importance in $M t b$ infection control; in HIV and TB co-infection there is continuous loss of CD4 T cells, leading to an advancement to active form of TB [20]. Most cases of TB infection and death prevail among men, but the TB burden is also high among women. In 2012, the South-East region and Western Pacific region accounted for about $58 \%$ of the world's TB cases. Also, the African region had nearly one quarter of the world's cases; India and China recorded the highest number of cases with $29 \%$ and $12 \%$ of the global total respectively [21]. In Sub-Saharan Africa, TB has increased markedly over the past two decades and there was a reported doubling of annual incidence from 173.6 to 351.7 per 100,000 population between 1990 and 2007 [22]. MDR-TB can be defined as TB found to be resistant to both rifampicin and isoniazid drugs with or without resistance to other anti-TB drugs [23]. MDR-TB is on the increase; extensively drug-resistant tuberculosis (XDR-TB) and totally drug-resistant tuberculosis have already been reported. These obstacles impose rising threats to tuberculosis control [2, 24, 25].

\section{Bacteriology}

In 1882, Robert Koch discovered Mtb, the intracellular pathogen [26]. Mycobacteria are non-motile, aerobic bacteria that have characteristics of acid fastness (Zeihl-Neelsen staining) because of mycolic acid enriched cell wall [27]. $M t b$ are rod-shaped bacilli [28]. The presence of a wide array of complex lipids and lipoglycans on the cell surface of $M t b$ make it unique among the bacterial pathogens [29]. The hydrophobic nature and the complexity of the cell wall, which is composed of the following: arabinogalactan, peptidoglycan, fatty acid (mycolic acids), and glycolipids layered on top of the plasma membrane critically cause $M t b$ to deceive the immune system of the host [30].

\section{Immune response to tuberculosis infection}

Tuberculosis infection is airborne, its cycle starts when a host inhales infectious airborne particles, usually of less than $5 \mu \mathrm{m}$ diameter, containing infectious pathogen [31]. Alveolar macrophages engulf mycobacteria when they enter the lung, where $M t b$ reproduces, and inhibit macrophage killing mechanisms. Despite the inhibitory influence of $M t b$, infected macrophages secrete chemokines and cytokines, leading to the recruitment and activation of many immune cell populations to the lung [32]. The innate immunity activation depends on recognition of $M t b$ components of the cell wall as mycolic acid, mannan, and peptidoglycans through toll-like receptors [33]. Mycobacterial antigen recognition, macrophages, and dendritic cell (DC) activation as well as other cells involved in innate immunity need tolllike receptors [34]. The bacilli is engulfed by macrophage through phagocytic receptors, of which the complement and mannose receptors play an important role [35]. The immune cells enclose the pathogenic bacteria in the first stage of tuberculosis infection, intracellular multiplication occurs, and the bacteria-overloaded cells may traverse the alveolar barrier, affecting other tissues and organs [36]. Survival of $M t b$ in macrophages is achieved by inhibiting acidification of the phagosomal complement and also by inhibition of the fusion of the phagosome with lysosomes [35]. The cell-mediated immunity is effectively involved in regulating $M t b$ limitation in granulomatous lesions of the lungs, usually without eliminating the bacteria that prevail in the latent stage [37]. Besides macrophages, it is understood that DCs also play a role as an important intracellular niche for Mtb [38]. Dendritic cells are key regulators of adaptive immunity and are potent antigen presenting cells [39]. Dendritic cells have the unique ability to migrate to draining lymph nodes from the site of infection and afterwards recruit $\mathrm{T}$ cells of infection where they effectively activate the acquired immune response [40].

Protective immunity against $M t b$ depends critically on T lymphocytes, due to its intracellular lifestyle [41]. In tuberculosis, cellular responses are the mediators of both pathogenesis and protection, which involves primarily interactions of phagocytes of macrophage lineage and lymphocytes [42]. Production of cytokines like interferon $\gamma$ (INF- $\gamma$ ) and tumour necrosis factor (TNF) establish protective immune responses against Mycobacterium tuberculosis infection; both cytokines activate macrophage toward Mtb control [43]. The cytokines play an essential role in controlling mycobacterium growth by expression of reactive nitrogen and oxygen [44]. In the presence of oxygen, an enzyme associated with macrophage functions in tuberculosis, called nitric oxide synthese- 2 , catalyses the metabolism of L-arginine into L-citrulline and nitric oxide, which takes part in killing the intracellular pathogen [45]. The $M t b$ immune response consists of great number of different cell kinds, including T cells, neutrophils, B-cells, and natural killer cells, and the roles played by CD4 T helper type 1 cells are the best understood [46]. Other interleukin (IL) producing $\mathrm{T}$ lymphocytes such as CD8 $\mathrm{T}$ lymphocyte and CD4 cells are likely take part in protective immunity 
[47]. After infection, $M t b$ stimulates both CD4 and CD8 $\mathrm{T}$ cells and other immune cells; secretion of INF- $\gamma$ dominates a strong type 1 immune response [48]. During adaptive immune response to $M t b$ infection, CD4 cells are the primary source of INF- $\gamma$, which are required for the survival of the host during both phases of acute and chronic infection [49]. The need for INF- $\gamma$ in immune protection of tuberculosis is well established both in animal models and in humans [50]. Interferon $\gamma$ is the key cytokine in humans and also in mice, the role of which is to activate the bactericidal actions in the host cell, macrophage [51]. It has been reported that individuals with genetic deficiency in the INF- $\gamma$ receptor are more likely to be infected with mycobacterial [52]. Several studies have revealed an increased susceptibility to mycobacterial diseases in INF- $\gamma$-deficient mice and also in humans having INF- $\gamma$ receptor abnormalities [38]. INF- $\gamma$ expresses protein peptides cathelicidin and defensin- $\beta 2$, which are delivered to $M t b$ phagosomes via vitamin D-dependent pathway [53], defensins, and a single cathelicidin, LL-37, which are major groups of host defensive peptides in humans. Susceptibility to infectious diseases including tuberculosis has been reported due to alteration in the synthesis of these molecules [53-55].

\section{Vaccines}

Currently, most tuberculosis vaccines under different stages of clinical trials are focused on either replacement of BCG or as a booster following vaccination with prime BCG [56]. Subsequently to the failure of MVA85A in the last two years, there has been no new, prominent TB vaccine entering clinical testing. There are $16 \mathrm{~TB}$ candidate vaccines in clinical testing, classified into priming vaccines, prime boosters, and immunotherapeutic vaccines [57]. Most of these candidates are subunit vaccines; selected antigens of $M t b$ are expressed using recombinant viral vectors or are administered in combination as protein/ adjuvant [58]. The developmental pipeline of new TB vaccines is shown in Table 1.

\section{Bacillus Calmette-Guérin}

BCG vaccine was first developed from a virulent strain [68]. Attenuation of the original BCG strain of $M$. bovis led to the establishment of a BCG vaccine resulting from subcultures in a media aimed at preserving its immunogenicity [69]. BCG play a protective role against $M t b$ because it induces CD4 (T helper type 1) and CD8

Table 1. The developmental pipeline trend for new vaccines against tuberculosis

\begin{tabular}{|c|c|c|c|c|}
\hline TB vaccine & Vaccine Type/Strategy & Phase & Sponsors & Reference \\
\hline MTBVAC & $\begin{array}{l}\text { Live-attenuated vaccine/ } \\
\text { priming vaccine }\end{array}$ & Phase I & $\begin{array}{l}\text { University of Zaragoza, Biofabri, } \\
\text { The Tuberculosis Vaccine Initiative (TBVI) }\end{array}$ & [59] \\
\hline VPM1002 & Recombinant live/prime & Phase I & $\begin{array}{l}\text { Max Planck, Vakzine Projekt management } \\
\text { GmbH, The Tuberculosis Vaccine Initiative } \\
\text { (TBVI) }\end{array}$ & [60] \\
\hline Ad5 Ag85A & $\begin{array}{l}\text { Viral-vectored vaccine } \\
\text { Prime booster }\end{array}$ & Phase I & $\begin{array}{c}\text { McMaster University, Supported by Tianjin } \\
\text { Cansino Biotech. Inc }\end{array}$ & {$[61]$} \\
\hline $\mathrm{M} 72+\mathrm{AS} 0$ & Protein and adjuvant/prime booster & Phase IIb & GlaxoSmithKline, Aeras & {$[62]$} \\
\hline MVA85A & $\begin{array}{l}\text { Attenuated Mycobacterium } \\
\text { tuberculosis strain }\end{array}$ & Phase I & $\begin{array}{l}\text { The Tuberculosis Vaccine Initiative } \\
\text { (TBVI), Zaragoza, Biofabri }\end{array}$ & {$[63]$} \\
\hline Crucell Ad35+ MVA85A & Viral vector/Prime booster & Phase I & Crucell, Oxford University, Aeras & {$[62]$} \\
\hline Hybrid 1 + IC31 & $\begin{array}{l}\text { Recombinant protein/ } \\
\text { Prime-boost }\end{array}$ & Phase I & $\begin{array}{l}\text { Statens Serum Institut/Tuberculosis } \\
\text { Vaccine Initiative/Intercell }\end{array}$ & {$[64]$} \\
\hline Hybrid 4 + IC31 & Recombinant and adjuvant & Phase I & $\begin{array}{c}\text { Statens Serum Institut (SSI), Tuberculosis } \\
\text { Vaccine Initiative (TBVI) }\end{array}$ & {$[65]$} \\
\hline Hybrid 56 + IC31 & Adjuvanted subunit/Prime-Boost & Phase II & Statens Serum Institut & {$[66]$} \\
\hline ChAdOx1 85A + MVA85A & Viral vector/Prime-boost & Phase I & Oxford University & {$[62]$} \\
\hline ID93 + GLA-SE & Adjuvanted subunit/Prime-Boost & Phase I & Infectious Diseases Research Institute & {$[66]$} \\
\hline DAR-901 & Mycobacterial-whole cell or Extract & Phase I & Darmouth, Aeras & {$[63]$} \\
\hline TB/FLU-041 & Viral vector/prime booster & Phase I & $\begin{array}{c}\text { Research Institute for Biological Safety } \\
\text { Problems }\end{array}$ & {$[63]$} \\
\hline Mycobacterium vaccae & Therapeutic/Boost, Post infection & Phase III & NIH, Aeras, Immodulon & {$[60]$} \\
\hline RUTI & $\begin{array}{l}\text { Immunotherapeutic/ } \\
\text { Fragmented MTB }\end{array}$ & Phase II & Archivel Farma & {$[67]$} \\
\hline
\end{tabular}


(T cell) responses [70]. BCG gives efficient protection against tuberculosis in new-borns, but does not provide prevention of latent infection or reactivation of TB in adults [71]. BCG is still used for children in many countries as part of the WHO expanded program on immunisation, but it is surrounded with controversy regarding its efficacy in protecting against adult and adolescent forms of TB [72]. BCG vaccine evaluation began formally in the 1930s, but scientists became aware of the degree of dissimilarity between the various results in the 1950s. In the UK, major trials carried out by the Medical Research Council indicated that more than $75 \%$ protection, whereas trials carried out by the US Public Health Services recorded less than $30 \%$ protection. Following trials and other studies resulted in a persistent broad range of estimates [73]. BCG has shown protective efficacy in adult pulmonary tuberculosis with reported variability in efficiency ranging from 0 to 80 per cent [74]. There is need of both Th1 and Th17 responses for an ideal host protection against tuberculosis; BCG happens to induce Th1 response and fails to induce Th17 response in the lung. The ability of BCG to induce Th1 but not Th17 mostly leads to inferior efficiency of BCG vaccine [75]. Interferon $\gamma$ is produced as a result of Th1 response I sand required for protection. Marchant et al. demonstrated that Th1 memory response is induced at birth upon BCG immunisation in a similar way when administered later in life [76]. An improved understanding of the reasons behind variability of BCG efficacy to such a great extent is important to assess new vaccines against tuberculosis, which are undergoing clinical trial [77]. The different levels of protection could be due to variations in BCG strain from different locations [77]. Immune response in individuals might be influenced by exposure to other environmental mycobacteria (EM), which, as a result, interfere with the effectiveness of BCG. Efficacy of BCG $>70 \%$ was reported in populations from nations situated far away from the equator having no or less prevalence of EM [78]. The effectiveness of BCG was found to be significantly lower in individuals of countries located near to the equator [79]. It has been reported that BCG has a protection time ranging from 10 to 20 years in the majority of cases [80]. Recently, Nguipdop-Djoma Patrick et al. demonstrated that BCG vaccination exhibited long-lasting protective ability against TB in individuals aged 12-50 years with negative tuberculin skin test and vaccination carried out between the years 1962 and 1975. Their results revealed that the rates of TB in unvaccinated and vaccinated participants are 3.3 and 1.3 per 1000 person-years, respectively [81]. BCG has been used in neonates since 1974 , and it provides protection against tuberculosis and tuberculosis meningitis with a $50 \%$ reduced risk of disease development in young children [82]. Despite being a safe vaccine, $B C G$ vaccines are considered among the most reactogenic vaccines, with reactogenicity depending on variation with different strains and the number of viable bacilli
[83]. Immunocompromised children infected with HIV or immunosuppressed individuals are especially vulnerable to complications of BCG vaccine [84]. A study was carried out on 349 BCG-immunised patients having severe combined immunodeficiency in 17 countries; the results indicated a high rate of complications of BCG vaccine [79].

\section{Live attenuated TB vaccines}

Improvement of the vaccine depends on strengthening the immunogenicity and persistence of a genetically modified recombinant strain of BCG (rBCG). Hence, a genetically manipulated $\mathrm{rBCG}$ could be more efficient compared to the parental BCG due to introduction of some parts of DNA (genes) lost during in vitro attenuation [85]. The loss of the RD1 region is the genetic principal behind BCG attenuation - the region encoding the machinery needed to synthesise and export the major T-cell antigen/virulence factor ESAT-6/CFP-10 [86]. The first recombinant BCG was generated by Horwitz et al. [87] and Horwitz and Harth [88]. rBCG3 overexpressed antigen Ag85b, which induced protection against TB significantly in animals. Compared with parental BCG, rBCG30 significantly increased Ag85b-specific T cells that inhibit intracellular mycobacteria [89].

VPM1002 is the second recombinant BGC vaccine candidate [79], formed because of two variations of live $M t b$. The gene encoding for Listeriolysin (Hly) from Listeria monocytogenes incorporated into the genome of $\mathrm{BCG}$ [59], rBCGUre:CHly*, conferred high protection against Mycobacterium tuberculosis challenge through aerosol. This improved protection was because of efficient perforation of the phagocyte phagosomal membrane by Listeriolysin (Hly) [90]. rBCGUre:CHly* is now in the phase of clinical trial due to its enhanced protection against tuberculosis [91]. Recombinant rBCGUre:CHly* constructs movement from endosomes to cytosol due to the activity of Listeriolysin with concomitant deletion of the urease gene. Loss of the urease gene leads to improved mycobacterial antigen processing via MHC I pathway as well as improved CD8 cytotoxic T cell activity [92].

BCG::ESAT-L28A/L29S improved BCG strain with modifications at amino acid residues. $\mathrm{Leu}^{28}{ }_{-} \mathrm{Leu}^{29}$ of the ESAT molecule showed strong attenuation in mice and high protective efficiency both in mouse and guinea-pig vaccination-infection models [93]. Chun Wang et al., 2012 [94] constructed three recombinant BCG strains that overexpressed immunodominant antigens of Mycobacterium tuberculosis, Ag85B (rBCG::85B) and Ag85A (rBC$\mathrm{G}:: 85 \mathrm{~A})$. Both recombinants (rBCG::AB) provided stronger and longer-lasting protection compared to the BCG containing vector without insert pMV261(rBCG::261) using mice.

In January 2013 MTBVAC entered a phase I clinical trial, and it is the live-attenuated Mycobacterium tuberculosis vaccine that entered the phase I trial. It is a derivative 
of attenuated strain SO2 obtained by insertion of a kanamycin-resistance cassette in the phoP (phoP is a transcription regulator) gene of $M t b$ transcription. Mutation of phoP causes a lack of expression of several genes, including ESAT6, a virulence factor [59]. In preclinical studies it was found that MTBVAC showed the same safety and biodistribution profiles as BCG and indicated superior protection [86]. The satisfactory safety of MTBVAC could be explained based on the following factors: lack of front-line lipids, loss of ESAT-6 expression, and down-expression of the PhoP regulon, essentially for pathogenicity and virulence of Mycobacterium tuberculosis [86]. Highly attenuated MTBVAC could be a potential vaccine for populations with high-risk immunosuppression, due to inactivation of an additional gene-generated repeated protein (Erp) [63].

\section{Subunit and viral vector-based vaccines}

Live-based vaccines are not products chosen by most manufactures because of safety considerations, especially in immunosuppressed individuals, and technical challenges regarding reproducibility [16]. The main reasons for developing a recombinant protein-based vaccine are as follows: they develop less reactogenicity and are considered more potent, safer, and better characterised vaccines [95]. Mtb secretes proteins during in vitro growth. One of the possible ways of improvement towards a tuberculosis vaccine would involve use of such secreted proteins. Some of these proteins are immunogenic; these proteins or their agreeing genes could serve as a major part of either a DNA-based vaccine or a subunit vaccine. Identification of antigens secreted in the culture fluid is important for establishing protective immune response against TB [85]. Several studies carried out have shown promising results for DNA vaccination against tuberculosis. DNA vaccines express different $M t b$ antigens; these include: Ag85A, Ag85B, ESAT-6, MTP-64, PstS-3, and 65kDa heat-shock protein. These proteins were all found to be effective in inhibiting the growth of $M t b$-infected mice [96]. We produced a potential peptide/protein vaccine (Myt272-3) from a clone contracted by shotgun cloning in the University of Malaya Molecular Bacteriology and Toxicology laboratory. The protein has an approximate molecular mass of $10.58 \mathrm{kDa}$, which conformed to the computed MW by EXPASY MW bioinformatics tool. Both protein blast and MALTI-TOF analysis indicated homology with phenolpthiocerol synthesis polyketide synthase I PpSA of Mtb. In silico analysis of the protein also indicated non-allegenicity and antigenicity of the query protein sequence, which serves as a good guide for the design of a vaccine against TB.

$\mathrm{Wu} \mathrm{Li}$ et al. reported a recombinant adenovirus (Ad5-CEAB) expressing Mtb antigens Ag85A, Ag85B, CFP10, and ESAT6 proteins combined in a mixture [97]. Ad5-CEAB resulted in a strong antigen-specific immune response as well as heightened humoral responses with a dramatically antigen-specific serum immunoglobulin (IgG).

\section{Viral vector}

Ad5Ag85A is a viral vectored adenovirus serotype 5 vector vaccine expressing Ag85A developed by Mcmaster University and supported by Tianjin CanSino Biotechnology Inc. The vaccine went through Phase I trial in 24 Canadian adults: 12 from BCG nadve and 12 from previously BCG-vaccinated, healthy adults. No vaccine-related serious adverse effects were recorded. Ad5Ag85A had immunogenicity in both groups with stimulation of polyfunctional T-cell responses, but found more effectively boosted CD4 and CD8 T-cell immunity in a group of previously-immunised subjects compared to a BCG-nadve group, which is reassuring for its further clinical development serving as a booster vaccine candidate after BCG priming [61].

A phase I trial involving MVA85A combined with Crucell Ad35 (Crucell Ad35 + MVA85A) was carried out among 40 adult participants at Oxford University [62]. Research Institute for Safety Problems and the Research Institute on Influenza, in Russia, developed a recombinant influenza vaccine called TB/FLU-04L, which is composed of influenza virus strain A/Puetro Rico/8/34 H1N1 and $M t b$ antigens Ag85A and ESAT6. A phase IIa trial is being planned for this vaccine candidate, and a phase I trial was completed [63]. ChAdOx1.85A is another adenovirus vaccine that expresses $M t b$ antigen $\mathrm{Ag} 85 \mathrm{~A}$; a phase I clinical study is currently testing the safety of ChAdOx1.85A vaccination along with infusion with MVA85A in adults vaccinated with BCG in the United Kingdom [62].

\section{Subunit adjuvant}

Adjuvants includes compounds and molecules/macromolecular complexes capable of boosting the potency and effective duration of specific immunological response to antigens [98]. The major hindrance in developing vaccines against bacteria has been attributed to a lack of adjuvant that adequately stimulates cell-mediated immunity [99]. It is therefore essential to administer subunit vaccines with an adjuvant to enhance immune responses to subunit vaccines. The adjuvants approved for human use include Aluminum salts, AS03/04 and MF59. They are primarily promoters of a humoral or Th2 rather than Th1 response [99].

Hybrid $1+$ IC31 is a subunit adjuvant vaccine developed by the Statens Serum Institute, TBVI, and Intacell. It is a hybrid of ESAT6 and Ag85B antigen with IC31, the components of the adjuvant system are oligodeoxynucleotide ODN1a and the cationic protein polyamino acid KLK [63]. Reither et al. [100] evaluated vaccine candidate H1/IC31 in 48 patients infected with HIV, and the results showed durable Th1 immune responses.

Hybrid 4 + IC31 vaccine has a fusion of Mtb antigens (Ag85B and TB10.4) with adjuvant IC31, owned by Valneva. In 2014, a three-arm, phase IIa study was announced by Aeras in order to determine the safety and immunogenicity of $\mathrm{H} 4+\mathrm{IC} 31$ and $\mathrm{BCG}$ revaccination in approximately 1000 BCG-immunised, non-HIV adolescent 
South Africans [62]. Hybrid $4+$ IC31 adjuvant vaccine effectively boosted and lengthened immunity induced by $\mathrm{BCG}$, leading to enhanced protection against $M t b$ due to domination of immune response by INF- $\gamma$, TNF- $\alpha$, IL- 2 or TNF- $\alpha$, IL-2, and CD4 cell [101].

Hybrid 56 + IC31, a protein adjuvanated vaccine composed of H56, a fusion protein consisting of Ag85B, ESAT6, and Rv2660c (latency-associated protein), incorporated with the adjuvant IC31 [59]. Hybrid $56+$ IC31 subunit adjuvant vaccine showed an ability to control the late-stage of TB infection, and it contains the latent stage of tuberculosis [102].

Thacher et al. evaluated M72/AS01 candidate vaccine [103] on 37 HIV-infected adults on cART (combination anti-retroviral therapy) in Switzerland in a ratio $3: 1: 1$ to vaccine, adjuvant (AS01), and saline placebo. The vaccine was found to be immunogenic with induction of persistent CD4 T-cell responses specific to M72.

The Infectious Disease Research Institute came up with the ID93 vaccine, which is the most recent tuberculosis vaccine entering clinical trials, and it was designed to target both forms of active and latent tuberculosis [47]. IDR93 is a protein/adjuvant vaccine that combined four novel sets of antigens including Rv2608, Rv3619, Rv3620, and Rv1813 in addition to the adjuvant (synthetic MPL formulated in a glucopyranosyl lipid stable emulsion). Preclinical studies with mice revealed that IDR93 vaccine is protective almost at BCG levels, and in guinea pigs a combination of ID93 and BCG reduced the mortality rate [47].

\section{Immunotherapeutic vaccines}

The main targets of therapeutic vaccine design are to prevent latent infection or to reduce the need of chemotherapy [104]. RUTI is one of the therapeutic vaccines made of detoxified, fragmented $M t b$ cells delivered in liposomes. A previous study revealed that RUTI showed efficacy in controlling latent form of TB infection in mice and guinea-pigs, inducing a combined Th1/Th2/Th3 polyantigenic response after a short period of chemotherapy [105]. Nell et al. [106] evaluated a RUTI vaccine in a placebo-controlled clinical trial involving 95 patients infected latently with TB. The RUTI vaccine showed its immunogenicity. A phase 1 clinical trial (randomised, placebo-controlled) of RUTI vaccine was carried out in Spain using healthy white males without history of tuberculosis infection and prior BCG vaccination, to find the tolerability and immunogenicity of four RUTI doses (ranging between $5 \mu \mathrm{g}$ and $200 \mu \mathrm{g}$ ). After completion of the trial in October 2008, the results showed that all the doses were tolerable, but moderate pain was noted with higher doses. Thirty-five days after vaccination four specific antigens were traced [58].

Mycobacterium vaccae was developed as an immunotherapeutic vaccine initially by inactivation of the whole cell strain of M. vaccae [107]. In a phase III trial, variable
INF- $\gamma$ and humoral responses were induced by Mycobacterium vaccae, according to CD4 T-cells count, HIV viral load, and previous TB treatment [59]. In Tanzania, vaccination with a multiple-dose series of Mycobacterium vaccae to HIV-positive adults immunised with BCG at childhood was associated with significant protection of HIV-associated TB. These results revealed that vaccination with $M$. vaccae, aimed at HIV-associated tuberculosis prevention, showed no detrimental effect on CD4 cells [108]. In 2001, M. vaccae was approved for sale in China for immune therapeutic purposes against TB. It appeared to provide a measurable improvement in some geographical settings but not in other places; the inconsistency led to doubts about its efficacy by some researchers [61].

\section{Conclusions}

Despite the emergence of new tuberculosis drugs and diagnoses, there is a need for new, safe, and effective vaccines to hasten the progress towards eradication of TB [109]. The trend of the tuberculosis vaccine development pipeline is in progress, with about 16 vaccine candidates at various stages of clinical trial. Despite several TB vaccines in phase, I, II and III of clinical trials, there is also a need to consider research on reverse vaccinology that involves computational analysis of proteome of the Mycobacterium tuberculosis in order to select new antigens that could serve as novel and more effective/efficient vaccines against tuberculosis. In addition to research development towards an improved vaccine for TB infection, mass campaigns on vaccination are needed in order to achieve the target of eradication of tuberculosis by the year 2050 .

The authors wish to thank the University of Malaya for supporting this research through PPP grants (Project no: PG238-2014B) RG064-12BIO and RG523-13HTM.

The authors declare no conflict of interest.

\section{References}

1. Yuen CM, Weyenga HO, Kim AA, et al. (2014): Comparison of trends in tuberculosis incidence among adults living with HIV and adults without HIV - Kenya, 1998-2012. Plos One 9: e99880.

2. WHO, Global Tubeculosis Report (2012): WHO, Geneva, Switzerland. 2012, WHO/HTM/TB/2012.6.

3. Theron G, Zijenah L, Chanda D, et al. (2014): Feasibility, accuracy, and clinical effect of point-of-care Xpert MTB/ RIF testing for tuberculosis in primary-care settings in Africa: a multicentre, randomised, controlled trial. Lancet 383 : 424-435.

4. Finnie RK, Khoza LB, van den Borne B, et al. (2011): Factors associated with patient and health care system delay in diagnosis and treatment for TB in Sub-saharan African countries with high burdens of TB and HIV. Trop Med Int Health 16: 394-411. 
5. Harries AD, Zachariah R, Corbett EL, et al. (2010): The HIV-associated tuberculosis epidemic - when will we act? Lancet 375: 1906-1919.

6. Müller B, Borrell S, Rose G, Gagneux S (2013): The heterogeneous evolution of multidrug-resistant Mycobacterium tuberculosis. Trends Genet 29: 160-169.

7. Russell DG, Barry CE, Flynn JL (2010): Tuberculosis: what we don't know can, and does, hurt us. Science 328: 852-856.

8. Hartman-Adams H, Clark K, Juckett G (2014): Update on latent tuberculosis infection. Am Fam Physician 89: 889-896.

9. Vandemoortele J (2004): Are the millennium development goals feasible? In: Targeting development: critical perspectives on the millennium development goals. Richard B, Howard W (eds.). Taylor \& Francis Group 124-144.

10. Devarajan S, Miller M, Swanson EV (2002): Goals for development: History, prospects, and costs. World Bank Policy Research Working Paper (2819).

11. WHO, Global Tubeculosis Report (2015): WHO, Geneva Switzerland. 2015. WHO/HTM/TB/2015.22.

12. Copin R, Coscollá M, Efstathiadis E, et al. (2014): Impact of in vitro evolution on antigenic diversity of Mycobacterium bovis bacillus Calmette-Guerin (BCG). Vaccine 32: 5998-6004.

13. Kaufmann SH (2014): Tuberculosis vaccine development at a divide. Curr Opin Pulm Med 20: 294-300.

14. Kaufmann SH, Hussey G, Lambert PH (2010): New vaccines for tuberculosis. Lancet 375: 2110-2119.

15. Galagan JE (2014): Genomic insights into tuberculosis. Nat Rev Genet 15: 307-320.

16. Girard MP, Fruth U, Kieny MP (2005): A review of vaccine research and development: tuberculosis. Vaccine 23: 57255731 .

17. Kakchapati S, Choonpradub C, Lim A (2014): Spatial and temporal variations in tuberculosis incidence, Nepal. Southeast Asian J Trop Med Public Health 45: 95-102.

18. WHO, Global Tuberculosis Report (2014): WHO, Geneva, Switzerland. 2014. WHO/HTM/TB/2014.08.

19. Modjarrad K, Vermund SH (2010): Effect of treating co-infections on HIV-1 viral load: a systematic review: Lancet Infect Dis 10: 455-463.

20. Ahmed A, Rakshit S, Vyakarnam A (2016): HIV-TB co-infection: mechanisms that drive reactivation of Mycobacterium tuberculosis in HIV infection. Oral Dis 22 (S1): 53-60.

21. WHO, Global Tuberculosis Report (2013): WHO, Geneva, Switzerland. 2013. WHO/HTM/TB/2013.11.

22. Stuckler D, Basu S, McKee M, Lurie M (2011): Mining and risk of tuberculosis in Sub-saharan Africa. Am J Public Health 101: 524-530.

23. Rosilawati ML, Yasmon A (2012): Detection of multidrug-resistant Mycobacterium tuberculosis directly from sputum samples of patients from Jakarta, Indonesia by radioisotope-based PCR-dot blot hybridization. Southeast Asian J Trop Med Public Health 43: 89-95.

24. Abubakar I, Zignol M, Falzon D, et al. (2013): Drug-resistant tuberculosis: time for visionary political leadership. Lancet Infect Dis 13: 529-539.

25. Udwadia ZF, Amale RA, Ajbani KK, Rodrigues C (2012): Totally drug-resistant tuberculosis in India. Clin Infect Dis 54: 579-581.

26. Luca S, Mihaescu T (2013): History of BCG vaccine. Maedica (Buchar) 8: 53-58.

27. Ryan K, Ray C (eds.) (2004): Sherris Medical Microbiology. $4^{\text {th }}$ ed. 2004, McGraw Hill.
28. Randall PJ, Hsu N, Quesniaux V, et al. (2015): Mycobacterium tuberculosis infection of the 'non-classical immune cell'. Immunol Cell Biol 93: 789-795.

29. Forrellad MA, Klepp LI, Gioffré A, et al. (2013): Virulence factors of the Mycobacterium tuberculosis complex. Virulence 4: 3-66.

30. Fukuda T, Matsumura T, Ato M, et al. (2013): Critical roles for lipomannan and lipoarabinomannan in cell wall integrity of mycobacteria and pathogenesis of tuberculosis. MBio 4: e00472-12.

31. Jones-López EC, Namugga O, Mumbowa F, et al. (2013): Cough aerosols of Mycobacterium tuberculosis Predict New Infection. A Household Contact Study. Am J Respir Crit Care Med 187: 1007-1015.

32. Monin L, Khader SA (2014): Chemokines in tuberculosis: The good, the bad and the ugly. Semin Immunol 26: 552558.

33. Matucci A, Maggi E, Vultaggio A (2014): Cellular and humoral immune responses during tuberculosis infection: useful knowledge in the era of biological agents. J Rheumatol 91: 17-23.

34. Saraav I, Singh S, Sharma S (2014): Outcome of Mycobacterium tuberculosis and Toll-like receptor interaction: immune response or immune evasion? Immunol Cell Biol 92: 741-746.

35. Behar SM, Martin CJ Booty MG, et al. (2011): Apoptosis is an innate defense function of macrophages against Mycobacterium tuberculosis. Mucosal Immunol 4: 279-287.

36. Ahmad S (2010): Pathogenesis, immunology, and diagnosis of latent Mycobacterium tuberculosis infection. Clin Dev Immunol 2011: 814943.

37. Kozakiewicz L, Phuah J, Flynn J, Chan J (2013): The role of B cells and humoral immunity in Mycobacterium tuberculosis Infection. In: The new paradigm of immunity to tuberculosis. Adv Exp Med Biol 783: 225-250.

38. Madan-Lala R, Sia JK, King R, et al. (2014): Mycobacterium tuberculosis impairs dendritic cell functions through the serine hydrolase Hip1. J Immunol 192: 4263-4272.

39. Lambrecht BN, Nety K, GeurtsvanKessel CH, Hammad H (2013): Lung dendritic cells and pulmonary defence mechanisms to bacteria. In: Mucosal immunology of acute bacterial pneumonia. Prince A (ed). 2013; 49-66.

40. Morris D, Gonzalez B, Khurasany M, et al. (2013): Characterization of dendritic cell and regulatory $\mathrm{T}$ cell functions against Mycobacterium tuberculosis infection. Biomed Res Int 2013: 402827.

41. Kaufmann SH (2013): Tuberculosis vaccines: time to think about the next generation. Semin Immunol 25: 172-181.

42. Mustafa AS, Al-Saidi F, El-Shamy AM, Al-Attiyh R (2011): Cytokines in response to proteins predicted in genomic regions of difference of Mycobacterium tuberculosis. Microbiol Immunol 55: 267-278.

43. Gopal R, Monin L, Slight S, et al. (2014): Unexpected role for IL-17 in protective immunity against hypervirulent Mycobacterium tuberculosis HN878 infection. Plos Pathog 10: e1004099.

44. Andersen P, Woodworth JS (2014): Tuberculosis vaccinesrethinking the current paradigm. Trends Immunol 35: 387395.

45. Duque-Correa MA, Kühl AA, Rodriguez PC, et al. (2014): Macrophage arginase-1 controls bacterial growth and pathology in hypoxic tuberculosis granulomas. Proc Natl Acad Sci 111: E4024-E4032. 
46. Ottenhoff T, Kaufmann S (2012): Vaccines against tuberculosis: where are we and where do we need to go. PLoS Pathog 8: e1002607.

47. Andersen P, Kaufmann SH (2014): Novel vaccination strategies against tuberculosis. Cold Spring Harb Perspect Med 4: a018523.

48. Nikolova M, Markova R, Drenska R, et al. (2013): Antigen-specific CD4-and CD8-positive signatures in different phases of Mycobacterium tuberculosis infection. Diagn Microbiol Infect Dis 75: 277-281.

49. Green AM, DiFazio R, Flynn JL (2013): IFN- $\gamma$ from CD4 $\mathrm{T}$ cells is essential for host survival and enhances CD8 $\mathrm{T}$ cell function during Mycobacterium tuberculosis infection. J Immunol 190: 270-277.

50. Desvignes L, Wolf AJ, Ernst JD (2012): Dynamic roles of type I and type II IFNs in early infection with Mycobacterium tuberculosis. J Immunol 188: 6205-6215.

51. Sharma S, Kalia NP, Suden P, et al. (2014): Protective efficacy of piperine against Mycobacterium tuberculosis. Tuberculosis 94: 389-396.

52. Wareham AS, Tree JA, Marsh PD, et al. (2014): Evidence for a role for interleukin-17, Th17 cells and iron homeostasis in protective immunity against tuberculosis in cynomolgus macaques. PloS One 9: e88149.

53. Andersen P, Woodworth JS (2014): Tuberculosis vaccinesrethinking the current paradigm. Trends Immunol 35: 387395.

54. Rivas-Santiago B, Castańeda-Delgado JE, Santiago CER, et al. (2013): Ability of innate defence regulator peptides IDR-1002, IDR-HH2 and IDR-1018 to protect against Mycobacterium tuberculosis infections in animal models. PloS One 8: e59119.

55. Rivas-Santiago B, Serrano CJ, Enciso-Moreno JA (2009): Susceptibility to infectious diseases based on antimicrobial peptide production. Infect Immun 77: 4690-4695.

56. Gopal R, Khader SA (2013): Vaccines against tuberculosis: moving forward with new concepts. Expert Rev Vaccines 12: 829-831.

57. Schito M, Migliori GB, Fletcher HA, et al. (2015): Perspectives on advances in tuberculosis diagnostics, drugs, and vaccines. Clin Infect Dis 61 (suppl 3): S102-S118.

58. Whole mycobacteria cell vaccines for tuberculosis summary group (2015): Developing whole mycobacteria cell vaccines for tuberculosis: Workshop proceedings, Max Planck Institute for Infection Biology, Berlin, Germany, July 9, 2014. Vaccine 33: 3047-3055.

59. Montagnani C, Chiappini E, Galli, L, de Martino M (2014): Vaccine against tuberculosis: what's new? BMC Infect Dis 14 (Suppl 1): S2.

60. Hawkridge T, Mahomed H (2011): Prospects for a new, safer and more effective TB vaccine. Paediatr Respir Rev 12: 46-51.

61. Ocampo M (2015): Vaccines-Recent advances and clinical trials. In: Tuberculosis-expanding knowledge. Wellman R (eds.). InTech 103-121.

62. Frick M (2015): The tuberculosis vaccines pipeline: A new path to the same destination? In: Pipeline report HIV, hepatitis $\mathrm{C}$ virus and tuberculosis drugs, diagnostics, vaccines, preventive technologies towards a cure and immune-based and gene therapies in development. Anderea B (eds.). HIV i-Base/Treatment action group. 163-178.

63. Ahsan MJ (2015): Recent advances in the development of vaccines for tuberculosis. Ther Adv Vaccines 3: 66-75.
64. Jefferys R (2012): The tuberculosis vaccine pipeline. HIV treatment bulletin. HIV i-Base. 251.

65. Kaufmann SHE, Hussey G, Lambert PH (2010): New vaccines for tuberculosis. Lancet 375: 2110-2119.

66. da Costa C, Walker B, Bonavia A (2015): Tuberculosis Vaccines - state of the art, and novel approaches to vaccine development. Intl J Infect Dis 32: 5-12.

67. Wingfield C and Jefferys R (2011): The Tuberculosis vaccine pipeline. In: Pipeline report HIV, hepatitis $\mathrm{C}$ virus and tuberculosis drugs, diagnostics, vaccines, preventive technologies. $2^{\text {nd }}$ edition. HIV i-Base/Treatment action group. 155-162.

68. da Costa AC, Nogueira SV, Kipnis A, Junqueira-Kipnis, AP: Recombinant BCG: innovations on an old vaccine. Scope of BCG strains and strategies to improve long-lasting memory. In: Significance of antegen and epitope specificity in tuberculosis. Juray I, Tom HMO (eds.). Front Immunol 2014; 5.

69. Norouzi S, Aghamohammadi A, Mamishi S, et al. (2012): Bacillus Calmette-Guérin (BCG) complications associated with primary immunodeficiency diseases. J Infect 64: 543-554.

70. Poyntz HC, Stylianou E, Griffiths KL, et al. (2014): Non-tuberculous mycobacteria have diverse effects on BCG efficacy against Mycobacterium tuberculosis. Tuberculosis 94 : 226-237.

71. Mirlekar B, Pathak S, Pathade G (2012): Mycobacterium tuberculosis: approach to development of improved strategies for disease control through vaccination and immunodiagnosis. Indian J Lepr 85: 65-78.

72. Deng Y, Bao L, Yang X (2011): Evaluation of immunogenicity and protective efficacy against Mycobacterium tuberculosis infection elicited by recombinant Mycobacterium bovis BCG expressing human Interleukin-12p70 and Early Secretory Antigen Target-6 fusion protein. Microbiol Immunol 55: 798-808.

73. Fine PE (1995): Variation in protection by BCG: implications of and for heterologous immunity. Lancet 346: 13391345.

74. Yuan W, Dong N, Zhang L, et al. (2012): Immunogenicity and protective efficacy of a tuberculosis DNA vaccine expressing a fusion protein of Ag85B-Esat6-HspX in mice. Vaccine 30: 2490-2497.

75. Samuchiwal SK, Tousif S, Singh DK, et al. (2014): A peptide fragment from the human COX3 protein disrupts association of Mycobacterium tuberculosis virulence proteins ESAT-6 and CFP10, inhibits mycobacterial growth and mounts protective immune response. BMC Infect Dis 14: 355 .

76. Marchant A, Goetghebuer T, Ota MO, et al. (1999): Newborns develop a Th1-type immune response to Mycobacterium bovis bacillus Calmette-Guerin vaccination. J Immunol 163: 2249-2255.

77. Mangtani P, Abubakar I, Ariti C, et al. (2014): Protection by $\mathrm{BCG}$ vaccine against tuberculosis: a systematic review of randomized controlled trials. Clin Infect Dis 58: 470-480.

78. Barreto ML, Pereira SM, Ferreira AA (2006): BCG vaccine: efficacy and indications for vaccination and revaccination. J Pediatr (Rio J) 82: s45-s54.

79. Principi N, Esposito S (2015): The present and future of tuberculosis vaccinations. Tuberculosis 95: 6-13.

80. Moliva JI, Turner J, Torrelles JB (2015): Prospects in Mycobacterium bovis Bacille Calmette et Guérin (BCG) vaccine diversity and delivery: Why does BCG fail to protect against tuberculosis? Vaccine 33: 5035-5041. 
81. Nguipdop-Djomo P, Heldal E, Rodrigues LC, et al. (2016): Duration of BCG protection against tuberculosis and change in effectiveness with time since vaccination in Norway: a retrospective population-based cohort study. Lancet Infect Dis 16: 219-226.

82. Bruffaerts N, Romano M, Denis O, et al. (2014): Increasing the vaccine potential of live $\mathrm{M}$. bovis BCG by coadministration with plasmid DNA encoding a tuberculosis prototype antigen. Vaccines 2: 181-195.

83. Bernatowska E, Wolska-Kuśnierz B, Pac M, et al. (2007): Clinical guidelines Risk of BCG infection in primary immunodeficiency children. Proposal of diagnostic, prophylactic and therapeutic guidelines for disseminated BCG based on experience in the Department of Immunology, Children's Memorial Health Institute in Warsaw between 1980-2006. Kardiol Pol 65: 1307-1311.

84. Pac M, Bustamante J, Buda P, et al. (2012): Disseminated Mycobacterium tuberculosis complex infection in a girl with partial dominant IFN- $\gamma$ receptor 1 deficiency. Centr Eur Immunol 37: 378-381.

85. Sarhan MA (2010): Tuberculosis vaccine. Saudi Med J 31: 9-13.

86. Arbues A, Aguilo JI, Gonzalo-Asensio J, et al. (2013): Construction, characterization and preclinical evaluation of MTBVAC, the first live-attenuated M. tuberculosis-based vaccine to enter clinical trials. Vaccine 31: 4867-4873.

87. Horwitz MA, Harth G, Dillon BJ, Masleša-Galić S (2000): Recombinant bacillus Calmette-Guérin (BCG) vaccines expressing the Mycobacterium tuberculosis 30-kDa major secretory protein induce greater protective immunity against tuberculosis than conventional BCG vaccines in a highly susceptible animal model. Proc Natl Acad Sci 97: 1385313858.

88. Horwitz MA, Harth G (2003): A new vaccine against tuberculosis affords greater survival after challenge than the current vaccine in the guinea pig model of pulmonary tuberculosis. Infect Immun 71: 1672-1679.

89. Hoft DF, Blazevic A, Abate G, et al. (2008): A new recombinant bacille Calmette-Guerin vaccine safely induces significantly enhanced tuberculosis-specific immunity in human volunteers. J Infect Dis 198: 1491-1501.

90. Moliva JI, Turner J, Torrelles JB (2015): Prospects in Mycobacterium bovis Bacille Calmette et Guérin (BCG) vaccine diversity and delivery: Why does BCG fail to protect against tuberculosis? Vaccine 33: 5035-5041.

91. Orme IM (2013): Vaccine development for tuberculosis: current progress. Drugs 73: 1015-1024.

92. Svenson S, Källenius G, Pawlowski A, Hamasur B (2010): Towards new tuberculosis vaccine. Hum Vaccin 6: 309-317.

93. Bottai D, Frigui W, Clark S, et al. (2015): Increased protective efficacy of recombinant BCG strains expressing virulence-neutral proteins of the ESX-1 secretion system. Vaccine 33: 2710-2718.

94. Wang C, Fu R, Chen Z, et al. (2012): Immunogenicity and protective efficacy of a novel recombinant BCG strain overexpressing antigens Ag85A and Ag85B. Clin Dev Immunol 568383.

95. Unnikrishnan M, Rappuoli R, and Serruto D (2012): Recombinant bacterial vaccines. Curr Opin Immunol 24: 337-342.

96. Yu D, Hu X, Cai X (2008): Efficient tuberculosis treatment in mice using chemotherapy and immunotherapy with the combined DNA vaccine encoding Ag85B, MPT-64 and MPT-83. Gene Ther 15: 652-659.
97. Li W, Deng G, Li M, et al. (2014): A recombinant adenovirus expressing CFP10, ESAT6, Ag85A and Ag85B of Mycobacterium tuberculosis elicits strong antigen-specific immune responses in mice. Mol Immunol 62: 86-95.

98. Reed SG, Bertholet S, Coler RN, Friede M (2009): New horizons in adjuvants for vaccine development. Trends Immunol 30: 23-32.

99. Ottenhoff TH, Doherty TM, van Dissel JT, et al. (2010): First in humans: a new molecularly defined vaccine shows excellent safety and strong induction of long-lived Mycobacterium tuberculosis-specific Th1-cell like responses. Hum Vaccin 6: 1007-1015.

100. Reither K, Katsoulis L, Beattie T, et al. (2014): Safety and immunogenicity of $\mathrm{H} 1 / \mathrm{IC} 31 \AA$, an adjuvanted TB subunit vaccine, in HIV-Infected adults with CD4+ Lymphocyte Counts Greater than 350 cells/mm3: A Phase II, multi-Centre, double-blind, randomized, placebo-controlled trial. PloS One 9: e114602.

101. Billeskov R, Elvang TT, Andersen PL, Dietrich J (2012): The HyVac4 subunit vaccine efficiently boosts BCGprimed anti-mycobacterial protective immunity. PLoS One 7: e39909.

102. Lin PL, Dietrich J, Tan E, et al. (2012): The multistage vaccine $\mathrm{H} 56$ boosts the effects of BCG to protect cynomolgus macaques against active tuberculosis and reactivation of latent Mycobacterium tuberculosis infection. J Clin Invest 122: 303-314.

103. Thacher EG, Cavassini M, Audran R, et al. (2014): Safety and immunogenicity of the M72/AS01 candidate tuberculosis vaccine in HIV-infected adults on combination antiretroviral therapy: a phase I/II, randomized trial. AIDS 28: 1769-1781.

104. Ruiz Manzano J, Vilaplana C (2014): Will we be treating tuberculosis with vaccines in the XXI Century? Arch Bronconeumol (English Version) 50: 373-374.

105. Cardona PJ (2006): RUTI: A new chance to shorten the treatment of latent tuberculosis infection. Tuberculosis 86 : 273-289.

106. Nell AS, D'lom E, Bouic P, et al. (2014): Safety, tolerability, and immunogenicity of the novel antituberculous vaccine RUTI: randomized, placebo-controlled phase II clinical trial in patients with latent tuberculosis infection. PloS One 9: e89612.

107. McShane H (2011): Tuberculosis vaccines: beyond bacille Calmette-Guérin. Philosophical transactions of the royal society B: Biological Sciences 366: 2782-2789.

108. von Reyn CF, Mtei L, Arbeit RD, et al. (2010): Prevention of tuberculosis in Bacille Calmette-Guérin-primed, HIV-infected adults boosted with an inactivated whole-cell mycobacterial vaccine. AIDS 24: 675-685.

109. Churchyard GJ, Snowden MA, Hokey D, et al. (2015): The safety and immunogenicity of an adenovirus type 35-vectored TB vaccine in HIV-infected, BCG-vaccinated adults with CD4(+) cell counts $>350$ cells/mm3. Vaccine 33: 1890 1896. 